\title{
Simulation in vitro des fermentations cæcales du lapin en fermenteur à flux semi-continu. II. Effet de la nature de l'inoculum
}

\author{
D Adjiri 1, M Bouillier-Oudot 1, F Lebas 2, M Candau 1 \\ ${ }^{1}$ École nationale supérieure agronomique de Toulouse, laboratoire de zootechnie \\ et de productions animales, 145, avenue de Muret, 31076 Toulouse Cedex; \\ 2 INRA, laboratoire de recherches sur l'élevage du lapin, BP 27, \\ 31326 Castanet-Tolosan Cedex, France
}

(Reçu le 8 janvier 1992; accepté le 30 juin 1992 )

\begin{abstract}
Résumé - Deux fermenteurs de type Rusitec ont été conduits dans des conditions identiques mais ensemencés à partir d'inoculum provenant soit de cæcum de lapins, soit de contenu de rumen de bovins. Le substrat de fermentation était un aliment pour lapin ayant subi un double traitement enzymatique par de l'amylase et de la pepsine. Le taux de disparition en $48 \mathrm{~h}$ des constituants du substrat (MO et NDF) est significativement plus élevé en présence d'inoculum de rumen $(+10 \%$ et $+15 \%$ respectivement). Le pH est similaire pour les 2 fermenteurs $(6,6)$. Après $6 \mathrm{j}$ d'adaptation, la quantité d'azote protéique produite par $24 \mathrm{~h}$ est semblable pour les 2 fermenteurs. La production d'AGV est légèrement plus élevée en présence de l'inoculum de rumen, mais les faciès fermentaires sont très différents. Dans le fermenteur ensemencé par du contenu de rumen, le pourcentage d'acide propionique est supérieur à celui de l'acide butyrique ( 25 vs $7 \%$ ). Par contre, en présence de contenu cæecal, la proportion de $C_{3}$ et de $C_{4}$ est en faveur de ce dernier $\left(C_{3} / C_{4}=0,81\right)$. En conséquence, seul l'inoculum cæcal de lapin a été en mesure de provoquer le faciès fermentaire caractéristique de l'espèce.
\end{abstract}

lapin / cæcum artificiel / inoculum / acide gras volatil

Summary - In vitro simulation of rabbit caecal fermentation in a semi-continuous flow fermentor. II. Effect of inoculum type. Two Rusitec fermentors were operated under identical conditions. One was seeded with an inoculum of rabbit caecal contents, and the other with bovine rumen contents. The fermentation substrate was rabbit feed that had been digested with amylase and pepsin. The substrate constituents (organic matter, OM and NDF) were lost in $48 \mathrm{~h}$ at a significantly higher rate in the presence of rumen inoculum (OM: $+10 \%, N D F:+15 \%)$. The pHs of the 2 fermentors were similar at $\mathrm{pH}$ 6.6. The fermentors produced similar amounts of protein nitrogen per $24 \mathrm{~h}$, after $6 d$ of adaptation. Volatile fatty acid production was slightly higher in the presence of rumen inoculum. The fermentor inoculated with rumen contents produced a higher percentage of propionic acid $(25 \%)$ than of butyric acid $(7 \%)$, while fermentation with rabbit caecal contents gave the opposite ratio $\left(C_{3} / C_{4}=0.81\right)$. Consequently, only the rabbit caecal inoculum provided the fermentation profile characteristic of the species. 


\section{INTRODUCTION}

Dans un article précédent (Adjiri et al, 1992) consacré à la simulation in vitro des fermentations cæcales du lapin, nous avons montré qu'il est possible de reproduire et de maintenir sur une période de 2 semaines, les caractéristiques principales des fermentations cæcales du lapin. Nous avons utilisé pour cela, un fermenteur à flux semi-continu, ensemencé à partir d'un inoculum constitué de contenu cæcal de lapin et alimenté par un aliment de type commercial préalablement traité par de l'amylase et de la pepsine.

Afin d'étudier l'importance de l'origine de l'inoculum dans le déterminisme des fermentations observées in vitro et de confirmer que l'obtention de ce faciès cæcal est bien lié à la nature de l'inoculum et non à la qualité du substrat utilisé, nous avons comparé les paramètres fermentaires de 2 fermenteurs conduits dans les mêmes conditions : l'un ensemencé avec du contenu cæcal de lapin et l'autre avec le contenu d'un rumen de bovin.

\section{MATÉRIEL ET MÉTHODES}

Les conditions opératoires sont identiques à celles déjà utilisées (Adjiri et al, 1992). Ne sont donc rappelés ici que les éléments essentiels.

\section{Préparation du substrat}

Le substrat est réalisé à partir d'un aliment de type commercial. Après broyage, l'aliment est soumis à 2 hydrolyses. L'aliment broyé est mis à incuber en présence d' $\alpha$-amylase $(8 \mathrm{~g}$ pour $100 \mathrm{~g}$ d'aliment et $500 \mathrm{ml}$ d'eau) pendant $24 \mathrm{~h}$ à $39^{\circ} \mathrm{C}$, sous agitation. Après acidification par de l'acide chlorhydrique jusqu'à pH 1,5, le substrat est de nouveau incubé avec $2 \%$ de pepsine pendant $4 \mathrm{~h}$ à $39^{\circ} \mathrm{C}$ sous agitation. Le $\mathrm{pH}$ est ensuite ramené à la neutralité avec de la soude, puis le résidu est filtré, lavé et séché.
Les traitements enzymatiques réduisent fortement la teneur en amidon : $1,4 \%$ contre $20,7 \%$ de la matière sèche dans l'aliment initial. Cette valeur est proche de celle du contenu iléal du lapin. La concentration en azote est réduite à $7,4 \%$ de la matière sèche contre $17,5 \%$ pour l'aliment initial. De manière à obtenir pour le substrat une proportion d'azote équivalente à celle du contenu iléal, une complémentation azotée journalière est effectuée moitié sous forme uréique dans la solution minérale et moitié sous forme de peptone de caséine introduite directement dans le fermenteur. Enfin, la teneur en fibres du substrat après traitement est supérieure à celle du contenu iléal de lapin : $69 \%$ NDF vs $46 \%$.

\section{Appareillage}

Le système de fermentation utilisé est identique au Rusitec mis au point par Czerkawski et Breckenridge (1977) pour la simulation de fermentations du rumen. Les 2 fermenteurs de 1 I de volume sont maintenus dans un bain-marie à $39^{\circ} \mathrm{C}$. L'approvisionnement en substrat des fermenteurs est effectué chaque jour. Une solution saline est apportée en continu $(0,5 \mathrm{l} / 24 \mathrm{~h})$. Les effluents liquides sont récupérés en vue d'analyse.

\section{Inoculum cæcal}

L'ensemencement est effectué à partir du contenu de 5 cæca de lapins nourris avec un aliment comparable à l'aliment expérimental. Le contenu des cæca est dilué par $300 \mathrm{ml}$ d'un mélange eau-solution minérale puis filtré sur de la gaze. Ensuite $500 \mathrm{ml}$ de filtrat et $100 \mathrm{~g}$ de résidu solide, placés dans un sachet nylon, sont introduits dans le fermenteur en même temps que $15 \mathrm{~g}$ du substrat placés dans un second sachet (fermenteur CAEC). Le volume est ensuite complété à $1 \mathrm{I}$.

\section{Inoculum de rumen}

Le contenu de rumen est prélevé sur 2 vaches porteuses de canule du rumen, nourries avec un 
régime à base d'ensilage de maïs et de foin de luzerne. Le jus de rumen est filtré sur de la gaze puis $500 \mathrm{ml}$ de filtrat et $100 \mathrm{~g}$ de résidu solide placés dans un sachet nylon sont introduits dans le fermenteur (fermenteur RUM) dans les mêmes conditions que pour l'inoculum cæacal.

\section{Fermentations}

Après ensemencement, un sachet de $15 \mathrm{~g}$ de substrat de fermentation est introduit tous les jours. Les sachets séjournent $48 \mathrm{~h}$ dans les fermenteurs, leur contenu est ensuite récupéré en vue d'analyse. La période d'étude dure 2 semaines.

\section{RÉSULTATS ET DISCUSSION}

Les fermentations obtenues à partir d'un ensemencement de contenu de rumen conduisent en $48 \mathrm{~h}$ à une dégradation de la matière organique et des constituants pariétaux du substrat significativement plus élevées que celles obtenues en présence de l'inoculum cæcal : respectivement $+9,7 \%$ et $+14,6 \%$ (tableau $\mathrm{I}$ ).

En dehors de la quantité d'azote microbien recueillie journellement dans l'effluent et de la production d'azote ammoniacal, les paramètres fermentaires présentent un profil relativement stable sur l'ensemble de la période d'étude. Le $\mathrm{pH}$ moyen $(6,6)$ n'est pas différent entre les 2 fermenteurs (tableau I).

Par contre, la quantité de l'azote protéique recueillie dans l'effluent décroît pendant les 5 premiers jours de fermentation pour le fermenteur ensemencé avec du contenu cæcal, alors qu'à partir de l'inoculum de rumen ce paramètre présente un profil stable (fig 1). Dans ce dernier cas, la concentration microbienne de l'inoculum liée à une teneur en matière sèche plus faible du contenu de rumen, est proportionnée à la quantité de substrat apportée, évitant ainsi le lessivage microbien de la période d'adaptation associé à l'inoculum

Tableau I. Influence de la nature de l'inoculum sur les paramètres de dégradation et fermentaires en caecum artificiel (moyenne et écart type de la moyenne).

\begin{tabular}{|c|c|c|c|}
\hline DMO \% & $51,74 \pm 2,79$ & $56,48 \pm$ & 2,47 \\
\hline DNDF \% & $41,24 \pm$ & $47,26 \pm$ & 2,93 \\
\hline $\mathrm{pH}$ & $6,61 \pm \quad 0,13$ & $6,59 \pm$ & 0,08 \\
\hline $\mathrm{N}$ prot $\left(\mathrm{mg} / \mathrm{j}^{\star}\right)$ & $173,90 \pm 116,3$ & $82,53 \pm$ & 23,7 \\
\hline $\mathrm{N}-\mathrm{NH}_{3}(\mathrm{mg} / \mathrm{j})$ & $227,61 \pm 40,52$ & $223,74 \pm$ & 31,72 \\
\hline AGV (mmol/ $)$ & $85,84 \pm$ & $90,37 \pm$ & 6,79 \\
\hline $\mathrm{C}_{2} \%$ & $65,25 \pm$ & $59,34 \pm$ & 1,14 \\
\hline $\mathrm{C}_{3} \%$ & $11,97 \pm$ & $24,87 \pm$ & 1,44 \\
\hline isoc $4 \%$ & $1,28 \pm$ & $1,43 \pm$ & 0,11 \\
\hline $\mathrm{C}_{4} \%$ & $15,04 \pm$ & $7,45 \pm$ & 1,22 \\
\hline isoC $_{5} \%$ & $2,13 \pm$ & $3,33 \pm$ & 0,47 \\
\hline$\sigma^{5}$ & $1,98 \pm$ & $2,99 \pm$ & 0,36 \\
\hline
\end{tabular}

\footnotetext{
- Comparaison de moyennes non réalisée en raison de la différence importante des écarts types des 2 moyennes.
}

a,b Sur une même ligne les moyennes affectées de la mème lettre ne sont pas différentes au seuil $P=0,05$. 


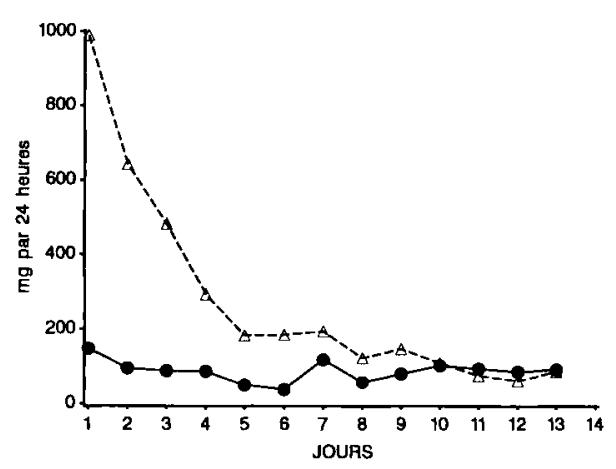

Fig 1. Évolution quotidienne de la production d'azote protéique $(\mathrm{mg} / \mathrm{j})$ recueillie dans les effluents de fermentation. Inoculum cæcal : triangles vides; inoculum de rumen : cercles pleins.

cæcal employé (Adjiri et al, 1992). Après cette période de $5-6 \mathrm{j}$, les 2 fermenteurs présentent des productions d'azote protéique équivalentes. Enfin, la production d'azote ammoniacal n'est pas significativement différente entre les fermenteurs (tableau I).

La quantité d'acides gras volatils produite journellement, légèrement plus élevée en présence de l'inoculum de rumen, est à mettre en rapport avec une dégradation plus importante du substrat. Cependant, cette différence n'est pas significative.

Les faciès fermentaires, étudiés à travers les proportions molaires des différents acides gras volatils produits, sont très différents entre les fermenteurs. Pour le fermenteur ensemencé avec le contenu de rumen le pourcentage d'acide propionique est supérieur à celui de l'acide butyrique (25 vs $7 \%$ ). Par contre, en présence de l'inoculum cæcal, le rapport $\mathrm{C}_{3} / \mathrm{C}_{4}$ reste en faveur de l'acide butyrique durant l'ensemble de la période de fermentation, en accord avec les données in vivo et nos données antérieures obtenues in vitro (Adjiri et al, 1992).
Par ailleurs, nous avons pu observer avec l'inoculum cæcal seulement, l'apparition de $\mathrm{C}_{6}$. Nous avons déjà souligné que Demaux et al (1981) ont observé la présence d'acide caproïque $\left(\mathrm{C}_{6}\right)$ chez des lapins conduits en alimentation rationnée. $\mathrm{La}$ conduite des fermenteurs semi-continus s'apparenterait à cette dernière pratique, mais cette production de $\mathrm{C}_{6}$ serait, en première analyse, spécifique de l'activité de la flore cæcale du lapin.

\section{CONCLUSION}

Même à partir d'un substrat s'apparentant au contenu iléal du lapin, I'utilisation d'une flore comme celle du rumen ne semble pas permettre d'obtenir un faciès fermentaire similaire à celui décrit dans le cæcum du lapin.

Ainsi, l'ensemencement des fermenteurs à partir du contenu cæcal de lapins est déterminant dans l'orientation des fermentations observées in vitro comme probablement in vivo, du fait de la spécificité de la flore microbienne de cette espèce.

\section{RÉFÉRENCES}

Adjiri D, Bouillier-Oudot $M$, Lebas $F$, Candau $M$ (1992) Simulation in vitro des fermentations cæcales du lapin en fermenteur à flux semicontinu. 1. Rôle du prétraitement du substrat alimentaire. Reprod Nutr Dev 32, 351-361

Czerkawski JW, Breckenridge G (1977) Design and development of a long-term rumen simulation technique (Rusitec). Brit J Nutr 38, 371-384

Demaux G, Guémon L, Gallouin F (1981) Temporal changes of some biochemical and histological parameters at different levels in the rabbit digestive tract. Reprod Nutr Dev 21, 868 